\title{
An Enhanced Cluster based Energy Efficient Optimal Routing in WSN
}

\author{
"G. Sai Krishna ${ }^{1}$, Dr. D. J. Nagendra Kumar ${ }^{2}$ \\ ${ }^{1}$ Department of CSE, Vishnu Institute of Technology, Bhimavaram, Andhra Pradesh, India \\ ${ }^{2}$ Professor, Department of IT, Vishnu Institute of Technology, Bhimavaram, Andhra Pradesh, India \\ *Corresponding Author : gsaikrishna255@yahoo.com
}

\begin{abstract}
Article Info

Volume 8, Issue 5

Page Number : 84-92

Publication Issue

September-October-2021

Article History

Accepted : 05 Sep 2021

Published : 14 Sep 2021

In recent years, Wireless sensor networks (WSNs) have been emerged as an important research area due to its wide spread application in various domains such as military sensing and tracking, environment monitoring, patient monitoring, etc. WSN also have various advantages in gathering the data also with data transmission as well. Even though WSN has such advantages, there is certain drawback related to the energy consumption for data transmission over the network. Wireless sensor networks basically depend on the availability of nodes for transmission and if some dead nodes are available on the designated path of transmission, there will be delay in communication and also will affect the energy consumptions. Also when a particular node is transmitting any data packet with high power, it may lead to interference which will affect the proper transmission of data and wastage of power as well. For power level reduction proper methodology has to be followed starting with the clustering and designing of routing protocols.

We intend to develop an enhanced clustering algorithm for initial clustering of sensor nodes for data transmission. Nodes will be clustered based on working attributes. Once nodes are clustered into different groups, transmission path will be assigned. An energy efficient optimal protocol will be designed in our approach for routing to improve the energy utilization by optimal power utilization. For optimization, we can employ multi objective optimization techniques which can enhance the optimal selection of power utilization. The proposed scheme will be then compared with some existing techniques to show the efficiency of the proposed approach.
\end{abstract}

Keywords: Wireless sensor network, Mobile node, Reference node, Routing protocols, TPSO, cluster, cluster head, energy-efficiency. 


\section{INTRODUCTION}

Wireless Sensor Network (WSN) is a network which establishes maximum number of sensor nodes which are situated in an application domain to observe the physical substances in an objective territory, for instance, temperature observing condition, water level, checking weight, and medicinal services, and different military applications. For the most part sensor nodes are furnished with self-bolstered battery control through which they can perform satisfactory activities and correspondence among neighboring nodes [1]. Expanding the lifetime of the Wireless Sensor systems, vitality preservation measures are basic for enhancing the execution of WSNs.

The best highlights of these WSNs incorporate little size, minimal effort, low calculation control, multifunctional (can perform detecting, information handling, steering, and so on.), and simple correspondence inside short separations. In unattended unfriendly locales, these gadgets are deployed that make the sensors hard to recharge. Be that as it may, different research works and strategies are completed for safeguarding vitality in sensor nodes to expand the system lifetime [2]. Drawn out system lifetime, dependable information exchange, vitality preservation in sensor nodes and versatility are the fundamental necessities for WSN applications. Due to the few requirements in the sensor nodes, WSN is having different issues, for example, inclusion region, and network lifetime.

Clustering conventions can fathom a portion of those issues because of their versatility, vitality effectiveness, and information conveyance dependability $[3,4]$. In a cluster based protocol, the network working time is isolated into rounds and each round is normally partitioned into three stages: Cluster Head $(\mathrm{CH})$ choice, group development, and information transmission.
Data delivery reliability is viewed as a key prerequisite in WSNs [5]. With the end goal to understand this prerequisite, cluster based protocols ought to embrace a multi-hop inter-cluster communication model as it is viewed as a more reasonable methodology due to the constrained transmission scope of the sensor nodes. A few connection quality-based cluster based protocols proposed so far utilize the separation between two nodes as a metric of their connection's quality. Nonetheless, a few investigations have demonstrated that separation is not really connected with connection quality as it disregards the connection asymmetry normal for WSNs [6].

The article is organised as follows: section 2 is composed of the recent works that are focused on sensor node routing process. Section 3 consists of the proposed design. Section 4 comprises the modelling parameters as well as the performance analysis of the proposed protocol with the other method and at last, section 5 is concluded with the overall study of the presented article.

\section{RELATED WORK}

In this section, the existing models that present the energy efficient routing of the mobile WSN that lacks the delivery rate, energy requirement, and robust network are presented.

Clustering strategies have been examined widely in the writing to enhance the efficiency of WSNs [7]. A substantial number of clustering based algorithms [811] dependent on heuristic strategies have been produced for WSNs. Among these LEACH [8] is an outstanding disseminated cluster based algorithm in which the sensor nodes themselves as a $\mathrm{CH}$ with some likelihood. LEACH gives critical vitality sparing and drags out the lifetime of the system contrasted with static grouping and least transmission vitality (MTE) convention. 
Hybrid energy-efficient distributed Clustering [12]is another dispersed cluster based protocol that is an expansion of LEACH. Clustering is accomplished with an iterative methodology. CHs choice in this convention is principally founded on the leftover vitality of every node. To build vitality productivity and further delay network lifetime, an auxiliary clustering parameter considers intra-group "communication cost" is presented which can be a component of neighbor closeness or cluster density.

In [13] the authors have proposed an "energy efficient proficient heterogeneous cluster scheme" for WSNs (EEHC) to think about the effect of heterogeneity of node as far as their efficiency in clustered network. They expected the situation where a level of the number of the populations in sensor nodes is outfitted with more vitality assets than the typical sensor hubs in the system. Three kinds of sensor nodes furnished with various efficiency levels were utilized. Nodes under first level are known as normal nodes, second level nodes are advanced node and third level nodes are super nodes. They demonstrated how the polling procedure of cluster heads ought to be adjusted suitably to manage heterogeneous nodes. The decision probabilities of $\mathrm{CHs}$ are weighted by the underlying efficiency of a node with respect to that of different nodes in the network.

A concentrated variant of (LEACH-C) is proposed in [14]. LEACH-C utilizes a Simulated Annealing (SA) way to deal with locate a foreordained number of $\mathrm{CHs}$ and to design the system into groups. The target work is characterized to limit the measure of vitality for the non- $\mathrm{CH}$ hubs to transmit their information to the $\mathrm{CH}$, by limiting the aggregate total of squared separations between all the non- $\mathrm{CH}$ and the nearest $\mathrm{CH}$.

Many researches examine about Fuzzy Logic (FL) how it very well may be connected on clustering with the goal that the energy consumption utilization will be limited. CHEF [15] considers two fuzzy parameters, for example, proximity distance and energy to elect the $\mathrm{CH}$. Abhijeet Alkesh et al. [16] and Taheri et al. [17] has considered three fuzzy parameters, for example, vitality, fixation, and centrality to compute the opportunity to be the $\mathrm{CH}$ and expands the network life time. In F-MCHEL [18], CH is chosen by using fuzzy standards dependent on energy and proximity of distance. The node is having most extreme lingering energy among the $\mathrm{CHs}$ is chosen as a Master Cluster Head (MCH) and sends the collected information to the base station. F-MCHEL is an enhanced version of CHEF. The points of interest and disservices of these conventions are talked about in [23]. In [19]- [22] numerous conventions have been talked about dependent on fuzzy procedures.

[24] address about extending the network lifetime by using two approaches: (1) relay nodes to mitigate network geometric deficiencies and using particle swarm optimization algorithm to find optimal sink position to make network energy efficient.

EBTAWSN (Energy Balanced Transmission Algorithm for Wireless Sensor Networks). The proposed approach can sense element of computation and communication, it also acts as an administrator to measure, reach and observe events in transmission energy in specific environment. In each of the transmission rounds nodes with remaining energy higher than threshold relays addition nodes and this distribution of energy load improves lifetime.

In [25] the only solution to maximize the lifetime of networks is by the reduction in the number of nodes involved as communication participants. The data aggregation based algorithms are played as most important power saving and reliable source of routing algorithms and this optimizes the communication via cluster head by the node degree considerations. Whatever algorithms help in minimizing the communication participants, the only way to maximize and improvise the life time of the network 
sensors are by the increase in sleeping nodes number with reduction in node ratio.

$\mathrm{CH}$-selection is an optimization problem which is NPhard in nature as described in [26]. Classical optimization algorithms are inefficient with the increasing size of the network. Particle swarm optimization (PSO) is one of the efficient nature inspired algorithms which can be a better choice for such NP-hard problem due to its ease of implementation, high quality of solution, ability to escape from the local optima and quick convergence.

$\mathrm{CH}$-choice is an optimization problem which is NPhard in nature as depicted in [26]. Conventional optimization algorithms are faulty with the expanding size of the network. Particle swarm optimization (PSO) is one of the effective nature enlivened algorithms which can be a superior decision for such NP-difficult issue because of its simplicity of usage, high caliber of solution, capacity to escape from the nearby optima and fast intermingling.

\section{PROPOSED METHODOLOGY}

In this article, the routing for the mobile WSN is presented by considering the certain factors of the network that tend to lose the energy of sensor node. By inducing the reference node on the network area the cluster and the cluster head are selected on then static network. Since the nodes are mobile nodes, none of the node will withstand a cluster so the number of nodes on cluster changes every time. The routing of the data from the mobile node to the base station is made by path exploration and optimal path selection process.

The system model consists of various parts that are required for the data transmission from one source to the receiver or base station. There are different types of clusters and each cluster consists of randomly placed nodes. The nodes which were at random distances are grouped to form the cluster by depending upon the distance between them. There will be a cluster head in each cluster which is used to transfer the data from one to the other. The source node in one of the cluster will produce the data which is to be transferred.

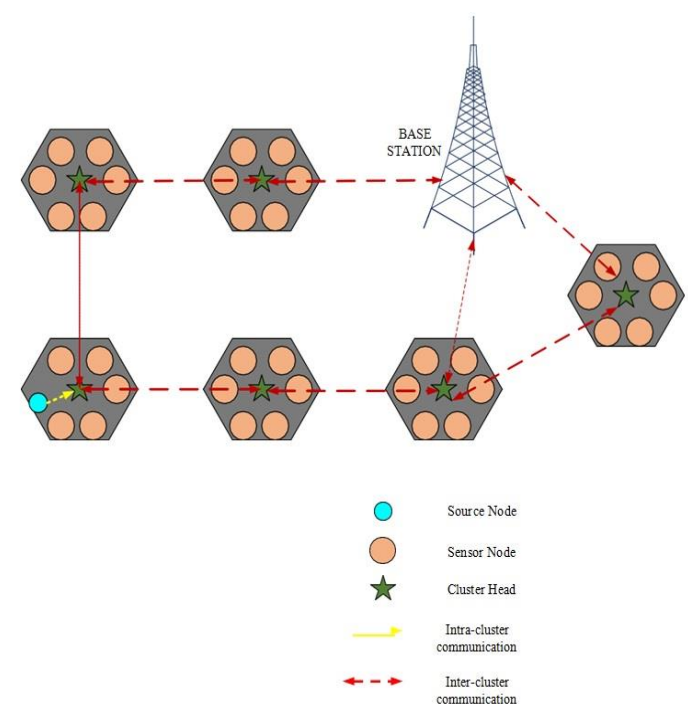

Figure 1 : State Model of the Network

A transmission power self-optimization (TPSO) technique able to adjust individually the transmission power of each sensor node that composes the WSN, in order to guarantee a predefined efficiency for the network [2ㅁ, 2ㅁ]. The TPSO technique aims at guaranteeing the lowest possible transmission power while maintaining the connectivity of the WSN as well as the reliability of the transmitted data. Thus, the main idea behind the self-optimization algorithm is to assure the trade-off between WSNs and data transmission energy consumption.

The top objective of this research is to maximise the lifetime of wireless sensor network and its nodes by rearranging connectivity between sensors. After implementing the TPSO algorithm, the process involves in three major processes namely construction of trees, Identifying critical tree using Prims 
algorithm and maximisation of lifetime of WSN using genetic algorithm through connecting networks. Prim's algorithm is a Greedy algorithm. It starts with an empty spanning tree. The idea is to maintain two sets of vertices. The first set contains the vertices already included in the MST, the other set contains the vertices not yet included. At every step, it considers all the edges that connect the two sets, and picks the minimum weight edge from these edges. After picking the edge, it moves the other endpoint of the edge to the set containing MST. Prim algorithm combines edge with least weight into spanning tree every time. So it can get spanning tree with least weight, namely MST. The algorithm allows finding the shortest path between the nodes, such that the data can be transferred from source node to destination node in less time and in an effective manner.

Construction of Tree: After the implementation, construction of tree processes will begin automatically. The base station sends to its neighbours the Identification parent and profounder. Every neighbour penetrates in the recursive schedule as well as identifies its neighbours; suppose any neighbour does not have profounder and origin of identification but it does not belong to the neighbour of base station, then neighbour penetrates in a schedule.

Identify the critical tree: When the tree's construction is completed; next processes begin naturally for identifying the critical tree that denotes a minimum ST (spanning tree) by adopting a greedy algorithm such as Prim's algorithm. The advantages of using Prim's algorithm are that the algorithm is relatively simpler and this algorithm is considerably faster in the limit when there exists a dense graph with more number of edges than vertices. The following is the pseudocode of prims algorithm.
In summary the Prim's algorithm operates in the following manner

- $\quad$ Choose any starting vertex.

- Look at all edges connecting to the vertex and choose the one with the lowest weight and add this to the tree.

- Look at all edges connected to the tree that do not have both vertices in the tree.

- $\quad$ Choose the one with the lowest weight and add it to the tree.

- Repeat step 2 until all vertices are in the tree.

Finally, Genetic Algorithm is applied in such a way that it increases the sensor nodes lifetime and networks lifetime. Genetic Algorithms are heuristic search algorithms based on the mechanism of biological evolution. Diversity in biological evolution is the variation of chromosomes between individual organisms [29]-[31]. This chromosome variation will affect the rate of reproduction and the level of the organism's ability to stay alive. The Genetic Algorithm uses the principle of finding neighborhood solutions based on natural selection and natural genetic mechanisms that can be used to solve optimization problems such as the Minimum Spanning Tree problem [32]-[34]. In the Genetic Algorithm, solving the Minimum Spanning Tree problem is almost the same as the solution to other optimization problems. The only difference is the chromosome coding process (encoding), weight calculation (decoding) and recombination or crossover. In addition to the case of the Minimum Spanning Tree, another step is added, namely the process of modifying the degree to check the presence of a circuit or not. The whole process will be experimented with the network of different size so that the performance of the proposed technique can be found. 


\section{RESULTS AND DISCUSSION}

In this section, the result analysis of the proposed model is presented. Here, on the network area of 100x100m 50 mobile sensor nodes are deployed. Since they are mobile a reference node is elected so as to make the data transmission. The communication radius of the reference node is $20 \mathrm{~m}$, thus the volume of the cluster is $40 \mathrm{~m}$. The reference node has the energy of 20J whereas the mobile nodes are set with the 2.5J of energy. The networking diagram of the model is shown in figure 4 .

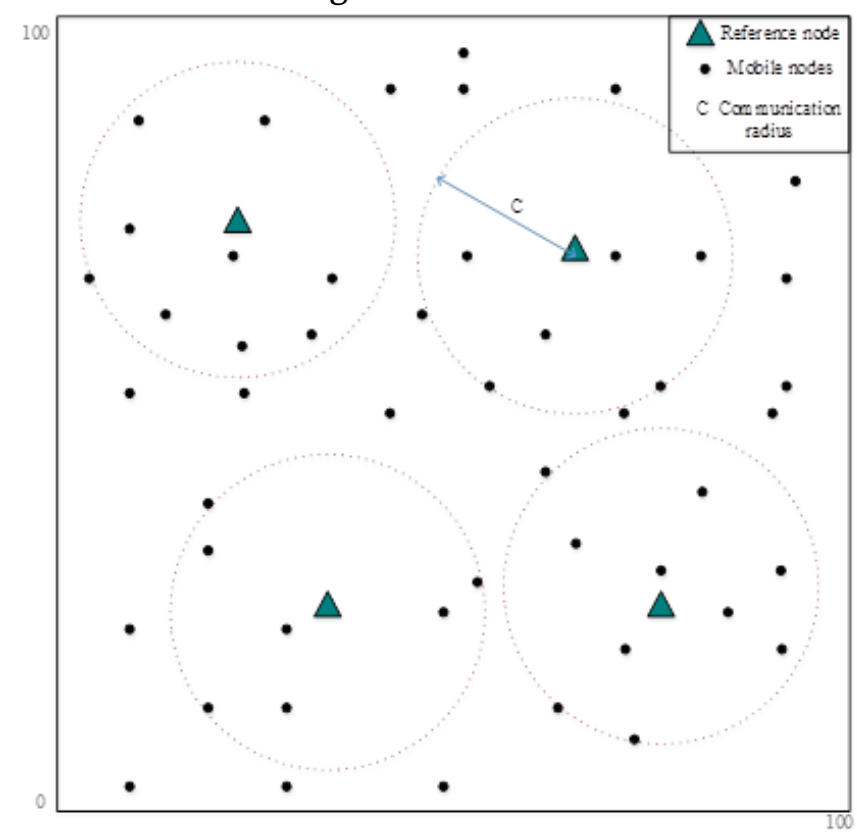

Figure 2: Network architecture diagram

\subsection{Performance analysis}

To analyse the performance of the proposed model, the parameters like energy consumption, packet transmission, delay, lifetime of the network is compared with the state-of-the-art methods.

\subsubsection{Energy Consumption}

Energy consumption gives the amount of energy drained by the sensor during the data transmission, here the energy loss due to mobility is also considered.

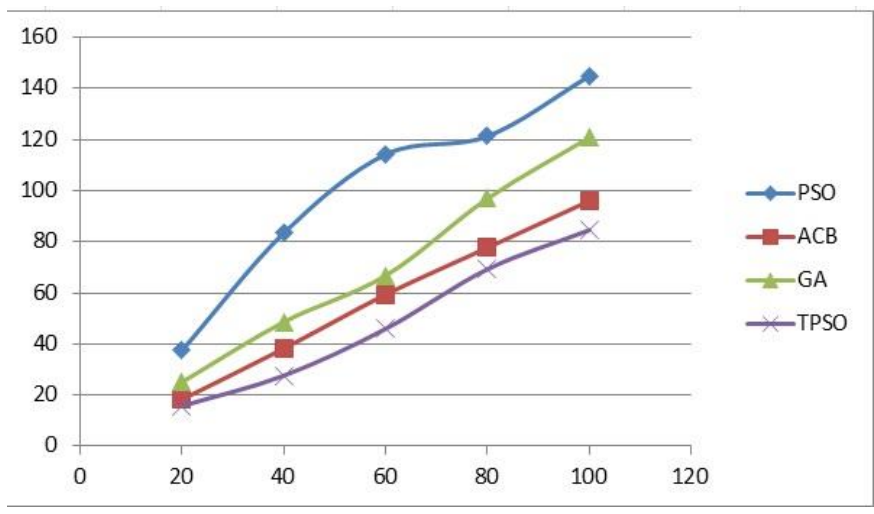

Figure 3: Energy consumption

The figure 3 gives the energy consumed by the network from overall energy of 145J is given. From total 145J of energy the proposed model have drained at 10000 rounds. The other methods have drained the total energy at earlier round.

\subsubsection{Packet transmitted to base station}

The total number of packet transmitted successfully to the base station is given in figure 4. On total of 10000 rounds $2.4 \times 10^{\wedge} 5$ packets are successfully transmitted by the proposed model.

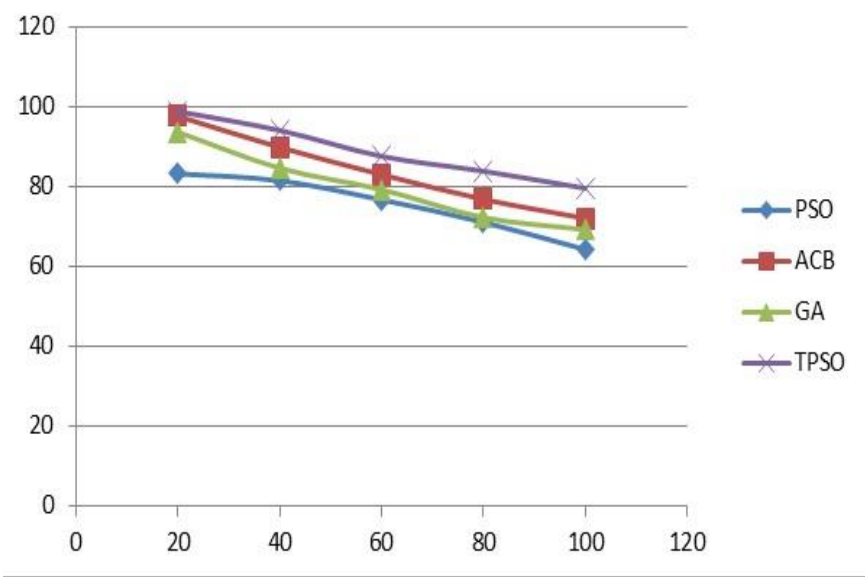

Figure 4: Packet Delivery ratio

The proposed model packet transmission rate is compared with the PSO, ACB, GA protocols. At 10000 rounds the existing methods have provided $0.07,0.34,0.67 \times 10^{\wedge} 5$ number of packets delivered, which is comparatively poor than the proposed model. 


\subsubsection{Network Life Time}

Delay is the average time required for the data to receive at the destination. Lifetime of a network depends on so many parameters like Energy consumption, packet delivery ratio, packet losses, etc.

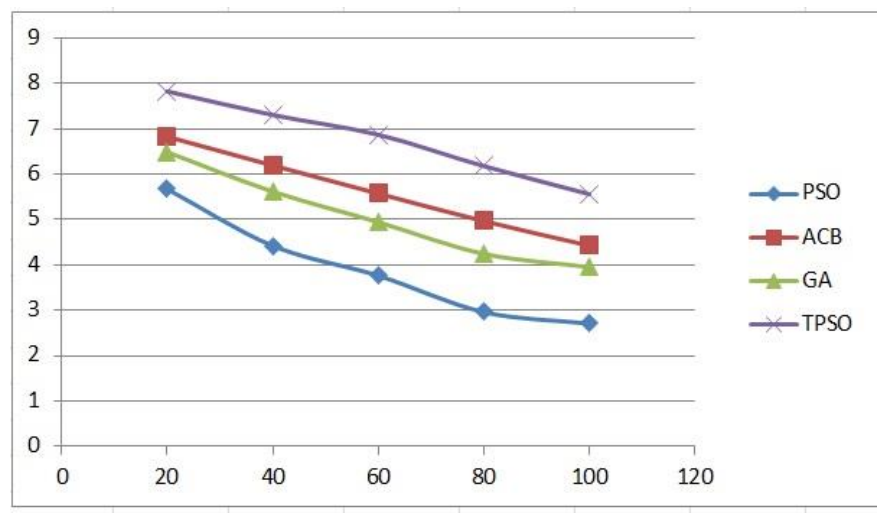

Figure 5: End-to-end delay

The Network life time is shown in the figure 5. The graph shows the Life time comparison of the proposed method with the PSO, ACB, GA. On the routing model the delay is analysed by varying the number of nodes on the network, since with increase in number of nodes the delay in the network is also increased. Even though the proposed model makes the exploration of multiple path, which increases with number of nodes, the delay is comparatively lower than other method and overall lifetime of the network also increases.

\section{CONCLUSION}

In this article, a novel protocol for energy-efficient routing of the mobile network is presented. The designed TPSO based protocol undergoes path exploration and path selection process by considering the congestion on the network, thereby reducing the retransmission rate which in turn reduces the energy used by the node. Along with the routing the presented model provides localization for the nodes. The performance of the proposed routing protocol compared with the state-of-the-art models. The presented TPSO based protocol consumed 60J of energy for 4000 rounds while the other methods have drained full of its energy on the same round. That is none of the node in the network is alive.

This research would help to create a WSN which would increase the nodes lifetime. Further, also we propose a methodology to find the optimal path between the nodes which also consequently helps to develop an enhanced clustering based approach in Wireless Sensor Networks with the aid of energy efficient optimal routing algorithm.

\section{REFERENCES}

[1]. Vimalarani, C., Subramanian, R. and Sivanandam, S.N., 2016. An enhanced PSObased clustering energy optimization algorithm for wireless sensor network. The Scientific World Journal, 2016.

[2]. G. Anastasi, M. Conti, M. Di Francesco, and A. Passarella, "Energy conservation in wireless sensor networks: a survey," Ad Hoc Networks, vol. 7, no. 3, pp. 537-568, 2009. View at Publisher - View at Google Scholar - View at Scopus

[3]. V. Sucasas, A. Radwan, H. Marques, J. Rodriguez, S. Vahid, R. Tafazolli, A survey on clustering techniques for cooperative wireless networks, Ad Hoc Networks 47 (2016) 53- 81. doi:https://doi.org/10.1016/j.adhoc.2016.04.008.

[4]. M. M. Afsar, M.-H. Tayarani-N, Clustering in sensor networks: A literature survey, Journal of Network and Computer Applications 46 (2014) 198 - 226.

[5]. M. A. Mahmood, W. K. Seah, I. Welch, Reliability in wireless sensor networks: A survey and challenges ahead, Computer Networks 79 (0) (2015) 166 - 187.

[6]. K. Srinivasan, P. Dutta, A. Tavakoli, P. Levis, An empirical study of low-power wireless, ACM Transactions on Sensor Networks 6 (2) 
(2010)

$16: 1-16: 49$. doi:10.1145/1689239.1689246.

[7]. V. Sucasas, A. Radwan, H. Marques, J. Rodriguez, S. Vahid, R. Tafazolli, A survey on clustering techniques for cooperative wireless networks, Ad Hoc Networks 47 (2016) 53 - 81.

[8]. Heinzelman, W. B., et al. (2000). Energy efficient communication protocol for wireless microsensor networks. In Proceedings of the 33rd Hawaii International Conference on System Sciences.

[9]. Xiang, L., et al. (2011). Compressed data aggregation for energy efficient wireless sensor networks. In 2011 8th Annual IEEE Communications Society Conference on Sensor, Mesh and Adhoc Communications and Networks (SECON) (pp. 46-54).

[10]. Liu, X. Y., et al. (2015). CDC: Compressive data collection for wireless sensor networks. IEEE Transactions on Parallel and Distributed Sysytems, 26(8), 2188-2197.

[11]. Xu, X., et al. (2015). Hierarchical data aggregation using compressive sensing (HDACS) in WSNs. ACM Transactions on Sensor Networks (TOSN), 11(3), 45.

[12]. Younis O, Fahmy S. HEED: a hybrid, energyefficient, distributed clustering approach for ad hoc sensor networks. IEEE Trans. Mob. Comput. 2004; 3(October (4)):366-79.

[13]. Kumar D, Aseri TC, Patel R. Eehc: energy efficient heterogeneous clustered scheme for wireless sensor networks. Comput. Commun. 2009;32(4):662-7

[14]. V. Sucasas, A. Radwan, H. Marques, J. Rodriguez, S. Vahid, R. Tafazolli, A survey on clustering techniques for cooperative wireless networks, Ad Hoc Networks 47 (2016) 53 - 81.

[15]. J.M. Kim, S.-H. Park, Y.-J. Han, and T. Chung, "CHEF: Cluster head election mechanism using fuzzy logic in wireless sensor networks," in Proc. ICACT, Feb. 2008, pp. 654-659.
[16]. A. Alkesh, A. K. Singh, and N. Purohit, “A moving base station strategy using fuzzy logic for lifetime enhancement in wireless sensor network," in Proc. Int. Conf. Commun. Syst. Netw. Technol., Jun. 2011, pp. 198-202.

[17]. H. Taheri, P. Neamatollahi, O. M. Younis, S. Naghibzadeh, and M. H. Yaghmaee, "An energy-aware distributed clustering protocol in wireless sensor networks using fuzzy logic," Ad Hoc Netw., vol. 10, no. 7, pp. 1469-1481, 2012.

[18]. T. Sharma and B. Kumar, "F-MCHEL: Fuzzy based master cluster head election leach protocol in wireless sensor network," Int. J. Comput. Sci. Telecommun., vol. 3, no. 10, pp. 8-13, Oct. 2012.

[19]. Z. W. Siew, C. F. Liau, A. Kiring, M. S. Arifianto, and K. T. K. Teo, "Fuzzy logic based cluster head election for wireless sensor network," in Proc. 3rd CUTSE Int. Conf., Miri, Malaysia, Nov. 2011, pp. 301-306.

[20]. V. Nehra, R. Pal, and A. K. Sharma, "Fuzzybased leader selection for topology controlled PEGASIS protocol for lifetime enhancement in wireless sensor network," Int. J. Comput. Technol., vol. 4, no. 3, pp. 755-764, Mar./Apr. 2013.

[21]. G. Ran, H. Zhang, and S. Gong, "Improving on LEACH protocol of wireless sensor networks using fuzzy logic,” J. Inf. Comput. Sci., vol. 7, no. 3, pp. 767-775, 2010.

[22]. H. Ando, L. Barolli, A. Durresi, F. Xhafa, and A. Koyama, "An intelligent fuzzy-based cluster head selection system for WSNs and its performance evaluation for D3N parameter," in Proc. Int. Conf. Broadband, Wireless Comput., Commun. Appl., Nov. 2010, pp. 648-653.

[23]. D. V. Puspalata and P. Nayak, "A clustering algorithm for WSN to optimize the network lifetime using type-2 fuzzy logic model," in Proc. 3rd Int. Conf. Artif. Intell., Modeling Simulations (AIMS), Kota Kinabalu, Malaysia, Dec. 2015, pp. 53-58. 
[24]. Dunuka. C and Para. U, (2014), Maximizing Lifetime For Wireless Sensor NetworkUsing Swarm Optimization And Energy-Balanced Nodes, International Journal of Scientific Research Engineering \& Technology (IJSRET), 3(6), pp. 1022-1027.

[25]. Lakshmi. J and Neelima. M, (2012), Maximising Wireless sensor Network life time through cluster head selection using Hit sets, IJCSI International Journal of Computer Science Issues, 9 ( 2,/3), pp. 328- 331.

[26]. Latiff, N. M. A., et al. (2007). Energy-aware clustering for wireless sensor networks using particle swarm optimization. In Proceedings of 18th Annual IEEE International Symposium on Personal, Indoor and Mobile Radio Communications (pp. 1-5).

[27]. F. Lavratti, A. R. Pinto, D. Prestes, L. Bolzani, F. Vargas, and C. Montez, "Towards a transmission power self-optimization in reliable wireless sensor networks," in Proceedings of the 11th Latin-American Test Workshop (LATW '10), March 2010.

[28]. F. Lavratti, A. R. Pinto, L. Bolzani et al., "Evaluating a transmission power selfoptimization technique for WSN in EMI environments," in Proceedings of the 13th Euromicro Conference on Digital System Design: Architectures, Methods and Tools (DSD '10), pp. 509-515, September 2010.

[29]. S. Gupta dan P. Panwar, "Solving Travelling Salesman Problem Using Genetic Algorithm," Int. J. Adv. Res. Comput. Sci. Softw. Eng., vol. 3, no. 6, hal. 376-380, 2013.

[30]. H. Tabatabaee, "Solving the Traveling Salesman Problem using Genetic Algorithms with the New Evaluation Function," Bull. Environ. Pharmacol. Life Sci., vol. 4, no. 11, hal. 124131, 2015.

[31]. Z. Ramadhan, A. Putera Utama Siahaan, dan M. Mesran, "Prim and Floyd-Warshall Comparative Algorithms in Shortest Path
Problem," in Proceedings of the Joint Workshop KO2PI and The 1st International Conference on Advance \& Scientific Innovation, 2018.

[32]. A. P. U. Siahaan, "Genetic Algorithm in Hill Cipher Encryption," Am. Int. J. Res. Sci. Technol. Eng. Math., vol. 15, no. 1, hal. 84-89, 2016.

[33]. A. Philip, A. A. Taofiki, dan O. Kehinde, "A Genetic Algorithm for Solving Travelling Salesman Problem," Int. J. Adv. Comput. Sci. Appl., vol. 2, no. 1, hal. 26-29, 2011.

[34]. Y.F. Waruwu, "Analisis Nilai Mutasi Dinamis pada Algoritma Genetika," Universitas Sumatra Utara, 2016.

\section{Cite this article as :}

G. Sai Krishna, Dr. D. J. Nagendra Kumar, "An Enhanced Cluster based Energy Efficient Optimal Routing in WSN", International Journal of Scientific Research in Science and Technology (IJSRST), Online ISSN : 2395-602X, Print ISSN : 2395-6011, Volume 8 Issue 4, pp. 84-92, September-October 2021. Available at doi : https://doi.org/10.32628/IJSRST2184114 Journal URL : https://ijsrst.com/IJSRST2184114 